\title{
Mixing in PCBM/P3HT bilayers, using in situ and ex situ neutron reflectivity
}

\author{
Dyfrig Môn and Anthony M. Higgins ${ }^{\text {a) }}$ \\ College of Engineering, Swansea University, Wales SA1 8EN, UK \\ Philipp Gutfreund \\ Large Scale Structures, Institut Laue-Langevin, Grenoble 38000, France \\ David James \\ College of Engineering, Swansea University, Wales SA1 8EN, UK
}

(Received 2 August 2016; accepted 30 January 2017)

\begin{abstract}
In situ and ex situ neutron reflectivity is used to characterize annealed regioregular-P3HT/PCBM bilayers. In situ annealing of a $20 \mathrm{~nm}$ PCBM/35 nm P3HT bilayer at $170{ }^{\circ} \mathrm{C}$ reveals rapid mixing of PCBM and P3HT to produce a polymer-rich layer that contains around 18-20\% PCBM. Samples with three different thicknesses of P3HT layer are ex situ annealed at $140{ }^{\circ} \mathrm{C}$. This again reveals migration of PCBM into the P3HT and vice versa, with the polymer-rich layer in the $20 \mathrm{~nm}$ PCBM/ $35 \mathrm{~nm}$ P3HT sample containing 19\% PCBM. Complete migration of the entire PCBM layer into the P3HT layer is observed for a $20 \mathrm{~nm}$ PCBM/80 nm P3HT bilayer. The robustness of fitted model composition profiles, in comparison with real-space imaging of sample surface morphology and previous work on annealed P3HT/PCBM bilayer compositions, is discussed in detail.
\end{abstract}

\section{INTRODUCTION}

Organic photovoltaic (OPV) devices offer the potential for low-cost and large-area solar cells. ${ }^{1}$ Ultrathin and flexible OPV devices also enable building and transport integration in products such as windows, transparent roofs, or car windshields. ${ }^{2,3}$ OPVs are particularly attractive because of the ability to manufacture them using high throughput printing processes. ${ }^{4}$ A popular choice for the active layer of OPVs is the bulk heterojunction (BHJ) formed by blending together electron-donating and electron-accepting species (either polymers or small molecules). A long-established and well-studied choice for such a blend consists of a fullerene derivative and a conjugated polymer. ${ }^{5,6}$ Here, the BHJ consists of a complex morphology that is designed to enable efficient separation of charges at the interface between the electrondonating polymer and the electron-accepting fullerene. The requirement for large interfacial area needs to be balanced by the provision of a continuous path for electron and hole transport. ${ }^{7}$ A full characterization and understanding of the morphology formed during device fabrication, and the potential evolution of structure/morphology during operation, ${ }^{8}$ is challenging because a number of different processes, such as phase separation, surface or

Contributing Editor: Dean DeLongchamp

a) Address all correspondence to this author.

e-mail: a.m.higgins@swansea.ac.uk

DOI: $10.1557 / \mathrm{jmr} .2017 .59$ substrate enrichment ${ }^{9}$ and crystallization (potentially of both components), ${ }^{2,6}$ may contribute to the final film structure and device performance. It is hoped that the development of further understanding of the morphology of OPV materials in thin-film architectures will underpin the successful commercial application of OPVs. ${ }^{2,5,10,11}$ This paper describes a study that focusses on conjugated polymer/fullerene mixing, by looking at the evolution of morphology within a simplified (bilayer) geometry, as a function of the polymer film thickness and temperature.

To understand the process controlling the morphology of fullerene and polymer OPVs, several studies on bilayers constructed from [6,6]-phenyl $\mathrm{C}_{61}$-butyric acid methyl ester (PCBM) and poly(3-hexylthiophene) (P3HT) have been carried out. ${ }^{11-20}$ These bilayers initially consisted of pure $\mathrm{P} 3 \mathrm{HT}$ and PCBM layers, with thermal annealing used as a means to study the intermixing of the PCBM and P3HT. A number of studies have shown that PCBM and P3HT can rapidly intermix,${ }^{11,14,18,19}$ and that this mixing involves diffusion of PCBM into amorphous regions of the P3HT. However, in PCBM/regioregular-P3HT bilayers, there are significant quantitative differences between the composition profiles in some of these studies. Treat et al. observed a uniform distribution of PCBM across $450 \mathrm{~nm}$ bilayers after $5 \mathrm{~min}$ of annealing at $150{ }^{\circ} \mathrm{C},{ }^{14}$ indicative of a PCBM loading of $40 \%$. In contrast, D. Chen et al., working in thinner bilayers but at similar annealing times and temperatures, observed only partial mixing, even though the PCBM constituted less than $40 \%$ of the total 
amount of material in the film. ${ }^{20}$ Both of these studies used dynamic secondary ion mass spectroscopy (DSIMS). Neutron reflectivity allows depth profiles to be determined with significantly higher resolution, and also allows for time-dependent (in situ) measurements. ${ }^{21,22}$ In addition to the measurement of layer composition offered by ion beam methods and ellipsometry, ${ }^{23,24}$ this increased resolution allows characterization of roughness at buried polymer interfaces, which is typically on the order of a few nanometers. ${ }^{25}$ Neutron reflectivity was performed on several P3HT/PCBM bilayers with different ratios of PCBM to P3HT by H. Chen et al. ${ }^{17}$ They reported only partial mixing in bilayers after annealing for $60 \mathrm{~min}$ at $150{ }^{\circ} \mathrm{C}$, a maximum loading of $\sim 22 \%$ PCBM in the P3HT-rich layer, and surface enrichment of PCBM within the P3HTrich (top) layer.

In this paper we look at the mixing between PCBM and regioregular P3HT as a function of film thickness and annealing temperature. We adopt the robust methodology that we have used in our previous studies of PCBM/ polymer bilayers $^{26}$ and use a combination of in situ annealing (annealing a sample in the neutron beam) and ex situ annealing (annealing a sample before measuring the reflectivity). Our results add to the strong body of evidence that there is rapid intermixing between PCBM and P3HT, and support the work of $\mathrm{H}$. Chen et al. ${ }^{17}$-giving a maximum loading of PCBM in P3HT of around $20 \%$. However, we find contrasting (and rather subtle) results in comparison with the reported findings of $\mathrm{H}$. Chen et al., in relation to PCBM-enrichment at the free surface.

\section{EXPERIMENTAL PROCEDURES}

\section{A. Materials}

PCBM, with 99.5\% purity, was obtained from Solenne BV (Groningen, The Netherlands). P3HT (weight-average molecular weight 36,000 , polydispersity index 1.8 , regioregularity 91-94\%) was supplied by Rieke Metals (Lincoln, Nebraska). Silicon [(100) with native oxide layer] was obtained from Prolog Semicor (Kiev, Ukraine). Mica sheets were obtained from Goodfellow (Huntingdon, United Kingdom). Toluene and chlorobenzene were purchased from Sigma-Aldrich (St. Louis, Missouri).

\section{B. Sample fabrication}

P3HT layers were spin-coated onto freshly-cleaved mica from toluene solutions $(0.1,1$, and $2 \mathrm{wt} \%$ for the $8 \mathrm{~nm}, 35 \mathrm{~nm}$, and $80 \mathrm{~nm}$ films respectively). PCBM layers were spin-coated from a chlorobenzene solution (1 wt \%) onto 2-inch diameter silicon substrates. A spin speed of 1000 revolutions per minute was used for all films. The spin-coated mica/P3HT layers and substrate/PCBM layers were then left for $24 \mathrm{~h}$ under vacuum before bilayer fabrication. Silicon/PCBM/P3HT bilayers were prepared by floating the $\mathrm{P} 3 \mathrm{HT}$ layer onto the surface of de-ionized water, and then depositing this layer onto the silicon/PCBM sample. Newly fabricated bilayers were firstly allowed to dry at ambient conditions and were then left under vacuum for $24 \mathrm{~h}$, before annealing.

\section{In situ annealing}

Samples were annealed on the neutron reflectivity beam-line, in a nitrogen atmosphere. The sample surface temperature during in situ annealing was calibrated by attaching an external thermocouple to the surface of duplicate silicon samples. There is a slight temperature overshoot (of a few ${ }^{\circ} \mathrm{C}$ ) at the start of in situ annealing, and the quoted temperature of $170{ }^{\circ} \mathrm{C}$, represents the approximate maximum sample surface temperatures reached during annealing. To keep this overshoot to a minimum the temperature was increased in two steps; an intermediate step from room temperature to $80{ }^{\circ} \mathrm{C}$, followed by a step from 80 to $170{ }^{\circ} \mathrm{C}$. A full reflectivity curve was measured at $80{ }^{\circ} \mathrm{C}$ (this took $90 \mathrm{~min}$ ) and the temperature was then raised to $170{ }^{\circ} \mathrm{C}$.

\section{Ex situ annealing}

Neutron reflectivity samples were annealed in the dark in a vacuum oven (Binder Inc., Bohemia, New York) with a vacuum of $\sim 10^{-3}$ Torr. Sample surface temperatures were calibrated by attaching an external thermocouple to the surface of duplicate silicon samples in the oven. At the end of annealing, samples were removed from the oven and rapidly quenched to room temperature on a metal surface. All quoted temperatures for ex situ annealed samples refer to the sample surface temperature. In contrast to the in situ annealing, there was no intermediate step here. The samples were heated to the annealing temperature in one go.

\section{E. Atomic force microscopy (AFM)}

Tapping mode was used on a Dimension-3100 (Veeco, New York, New York) or a CE100 (Park Systems Corporation, Suwon, South Korea), with OTESPA (Bruker Corporation, Billerica, Massachusetts) cantilevers to measure sample surface morphology and to determine initial bilayer film thicknesses. Film thickness was determined by scratching the surface of a single layer on a silicon substrate and scanning across the step edge of the scratch.

\section{F. Neutron reflectivity}

Measurements were carried out using beam-line D17 at the Institut Laue-Langevin (ILL) in time-of-flight (TOF) mode. ${ }^{27}$ TOF uses a broad range of neutron wavelengths at a fixed incident angle. At D17 two incident angles were used to cover the full reflectivity range, down to a reflectivity of approximately $10^{-6}$. Specular reflectivity was extracted using the program COSMOS within the Large Array Manipulation Program (LAMP) distributed by the ILL. Two incident-angles $\left(0.6\right.$ and $\left.2.4^{\circ}\right)$ were used to produce a full reflectivity curve, with resolution 
ranging from $2.3 \%$ at low momentum-transfer $(q)$, to $4.9 \%$ at high $q$. Measurements during in situ annealing used a single angle of $1^{\circ}$, but with chopper settings that gave a higher flux of neutrons. This angle just captured the critical edge for the sample, gave a lower $q$ range and a lower resolution (between 4.1 and $8.1 \%$ ), but the higher flux allowed much shorter acquisition-times. Samples were exposed to the neutron beam for acquisition-times of $30 \mathrm{~min}$ at $0.6^{\circ}, 60 \mathrm{~min}$ at $2.4^{\circ}$ and in $30 \mathrm{~s}$ time-slices at $1^{\circ}$.

Reflectivity was fitted using Parratt 32 and Motofit. ${ }^{28}$ Each layer was characterized by a thickness and scattering length density (SLD), plus an interfacial roughness between adjacent layers $(\sigma)$. The instrumental resolution was included within the models for all samples, at the values calculated from the slit widths and chopper settings. Except where stated otherwise, samples were fitted using fixed silicon and native silicon oxide layer parameters. The silicon substrate SLD was fixed at $2.07 \times 10^{-6} \AA^{-2}$. The silicon substrate $\sigma$ was fixed at $0.7 \mathrm{~nm}$. The native silicon oxide layer parameters were fixed at the following values; SLD $=3.48 \times 10^{-6} \AA^{-2}$, thickness $=1.5 \mathrm{~nm}$, and $\sigma=0.7 \mathrm{~nm}$.

SLDs for pure P3HT and PCBM were first calculated using the National Institute of Standards and Technology (NIST) SLD calculator ${ }^{29}$ with literature density values. ${ }^{14,17,30}$ The calculated value for PCBM was $4.4 \times$ $10^{-6} \AA^{-2}$ and for P3HT was $0.62 \times 10^{-6} \AA^{-2}$. These values were used as initial guesses when fitting the reflectivity curves of unannealed bilayers and ex situ annealed bilayers. For the in situ sample, the fit parameters from the previous time slot in the kinetic series were used as the initial guesses.

The percentage (by volume) of PCBM in the each layer was calculated using:

$$
100 \times \frac{\left(\mathrm{SLD}^{\text {layer }}-\mathrm{SLD}^{\text {pure P3HT }}\right)}{\left(\mathrm{SLD}^{\text {pure PCBM }}-\mathrm{SLD}^{\text {pure P3HT }}\right)},
$$

where $\mathrm{SLD}^{i}$ is the SLD of layer $i$, and the SLDs of pure P3HT and pure PCBM were set to the above calculated values.

\section{G. Optical microscopy}

Images were obtained using a Nikon Eclipse-E600FN microscope (Nikon, Tokyo, Japan), using a $50 \times$ objective.

\section{RESULTS AND DISCUSSION}

The reflectivity data from a bilayer measured at $80{ }^{\circ} \mathrm{C}$ and the corresponding fit obtained using a two-layer model are shown in Fig. 1(a). Figure 1(b) shows the fitted SLD profile. The SLD of the PCBM layer is $4.30 \times 10^{-6} \AA^{-2}$, and the SLD of the P3HT layer is $0.62 \times 10^{-6} \AA^{-2}$. These values correspond reasonably closely to previously reported values. ${ }^{17,31}$ The interfacial roughness, $\sigma$, between the two layers is $1.2 \mathrm{~nm}$. This SLD profile corresponds closely to that of an as-fabricated PCBM/polymer bilayer (see e.g., Môn et $\mathrm{al}^{26}$ ), and shows that little (if any) interdiffusion has occurred during the temperature step from room temperature to $80{ }^{\circ} \mathrm{C}$. We note that the data shown in Fig. 1 clearly has significantly more pronounced fringes in the reflectivity and a sharper interface than the unannealed PCBM/P3HT bilayer (with a $15 \mathrm{~nm}$ PCBM layer) fabricated by H. Chen et al. (Figs. 1 and 2). ${ }^{17}$

The sample from Fig. 1 was then annealed in situ for $30 \mathrm{~min}$. In situ annealing allows the reflectivity to be modeled from a known starting composition-profile, from which the subsequent SLD profile evolution can be followed in small steps. The sample temperature measurement in Fig. 2 shows that there was an initial period of approximately $5 \mathrm{~min}$, during which the sample temperature increased from 80 to approximately $170{ }^{\circ} \mathrm{C}$, followed by a $10 \mathrm{~min}$ period of temperature stabilization and a $15 \mathrm{~min}$ period of annealing at a constant temperature. The sample was then allowed to cool. Once the sample had again reached $80{ }^{\circ} \mathrm{C}$, another full reflectivity curve was measured.

The reflectivity data, fits and SLD profiles for the sample are shown in Fig. 3. The data is well-fitted using a simple bilayer model (i.e., two layers of uniform composition, separated by an interface parameterized by Gaussian roughness ${ }^{25,32,33}$ ) at all stages of annealing. Figure 3(a) shows how the reflectivity from this $35 \mathrm{~nm}$ P3HT bilayer sample changed with annealing. There is a clear change in the periodicity of the Kiessig fringes, indicative of significant changes in the composition profile within the sample. Figure 3(b) shows the SLD profile normal to the substrate for various annealing times and Fig. 4 shows the evolution of some of the fit parameters during annealing. In comparison to the composition profile at $80{ }^{\circ} \mathrm{C}$, the SLD profile progressed in three clear ways during annealing at $170{ }^{\circ} \mathrm{C}$; (i) the bottom layer SLD reduced; (ii) the top layer SLD increased; (iii) the interface roughness between the top and bottom layers increased. Small changes may be attributed to thermal expansion/contraction during heating/ cooling (see Leman et al. Supplementary Material ${ }^{23}$ ). However, this would only account for approximately a $1.5 \%$ change in SLD between 80 and $170{ }^{\circ} \mathrm{C}$. Systematic changes in the composition profile are complete within $15 \mathrm{~min}$, or possibly earlier. The ability to discriminate more precise kinetics in the time period before $15 \mathrm{~min}$ is confounded by scatter in the fit parameters, potential correlations between fit parameters (given the restricted $q$-range, lower resolution, and poorer statistics of the $30 \mathrm{~s}$ time-slices in comparison to the full reflectivity curves), and sample surface temperature stabilization. Scatter in the fit parameters is most evident for the top layer SLD. This is likely to be a result of the fact that the SLD of this layer is much lower than the 

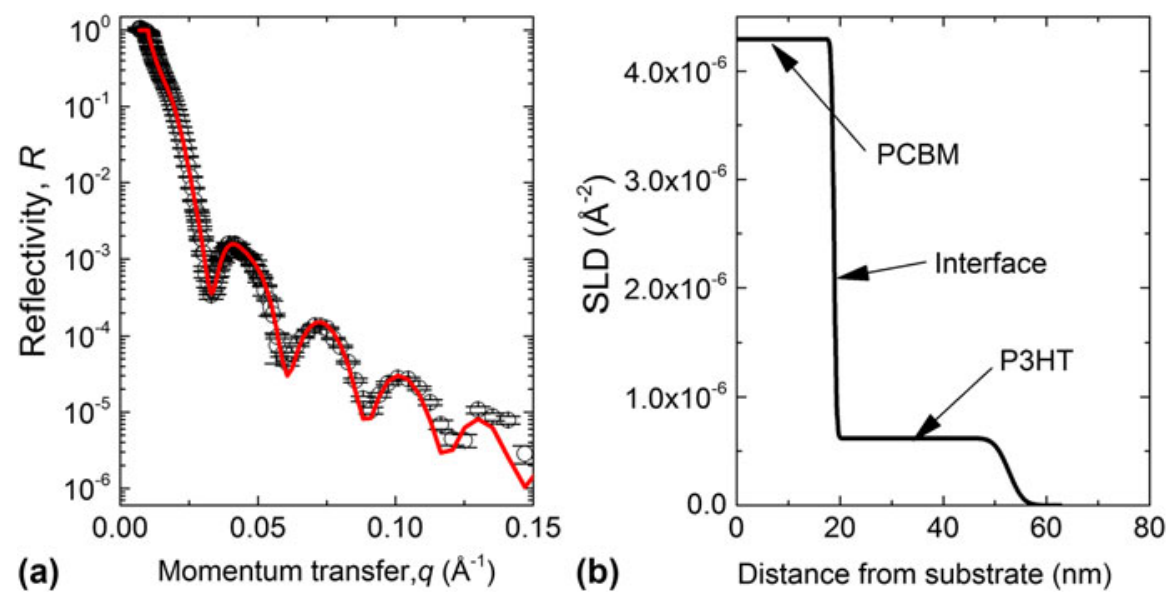

FIG. 1. (a) Reflectivity versus momentum transfer normal to the substrate $(q)$, and fit for an unannealed PCBM $(\sim 20 \mathrm{~nm}) / \mathrm{P} 3 \mathrm{HT}$ ( $\sim 35 \mathrm{~nm})$ bilayer on silicon. (b) The SLD profile corresponding to the best-fit line in (a). Fits were obtained using a bilayer model in which the thickness, SLD and roughness of each layer was allowed to vary.

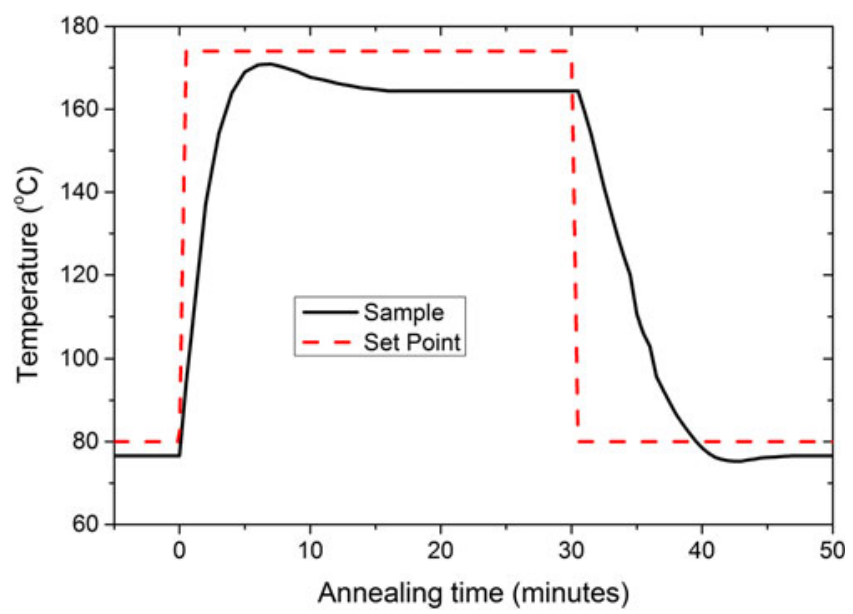

FIG. 2. Temperature versus in situ annealing time, for a nominal temperature of $170{ }^{\circ} \mathrm{C}$. The dashed line shows the set-point temperature and the continuous line shows the sample surface temperature.

bottom layer, resulting in the SLD of this layer having less of a constraining influence on the model reflectivity than that of the bottom layer (variation in the SLDcontrast at the interface, of a given magnitude, is a much larger fraction of the SLD of the top layer in comparison to the bottom layer). Figures 3 and 4 also show the changes in the composition profile obtained from fits of the full reflectivity curves before and after annealing. This data shows a difference in the fitted bottom layer SLD after annealing $\left(\sim 3.7 \times 10^{-6} \AA^{-2}\right)$ in comparison to the fit during annealing $\left(\sim 3.2 \times 10^{-6} \AA^{-2}\right)$. Given the differences between the quality of the measurements during and before/after annealing discussed above it is entirely possible, indeed likely, that this apparent change is a measurement artifact. However, in comparison with the SLD profile before annealing, the SLD profile
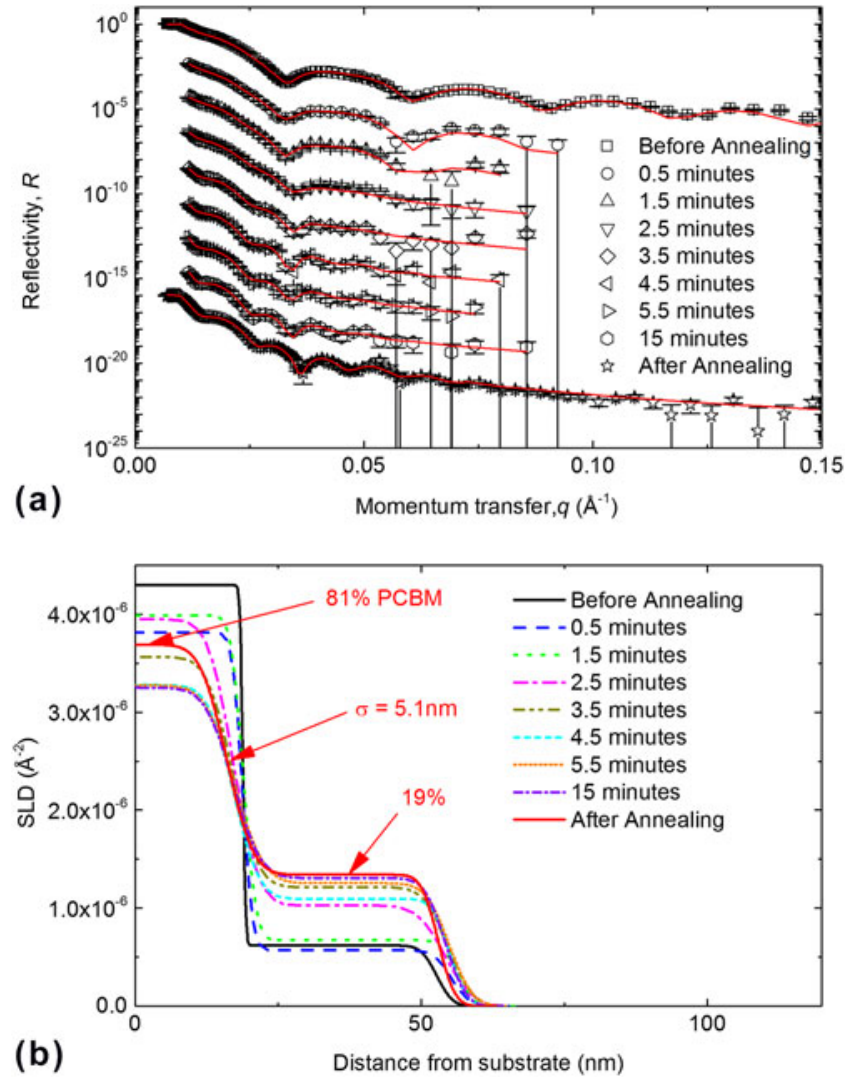

FIG. 3. (a) Neutron reflectivity data and (b) SLD profiles from a PCBM $(\sim 20 \mathrm{~nm}) / \mathrm{P} 3 \mathrm{HT}(\sim 35 \mathrm{~nm})$ bilayer annealed in situ at $170{ }^{\circ} \mathrm{C}$. Reflectivity curves are offset vertically for clarity. The lines in (a) are bilayer fits to the experimental data. The percentage PCBM in each layer and interface roughness, $\sigma$, are shown for the SLD profile after annealing. The measurements before-annealing and after-annealing were performed at $80{ }^{\circ} \mathrm{C}$. The times given in the legend are those at the end of each $30 \mathrm{~s}$ time slice.

after-annealing confirms the overall trend in the layer SLDs and interfacial roughness found during annealing. The composition profile after annealing has bottom and 

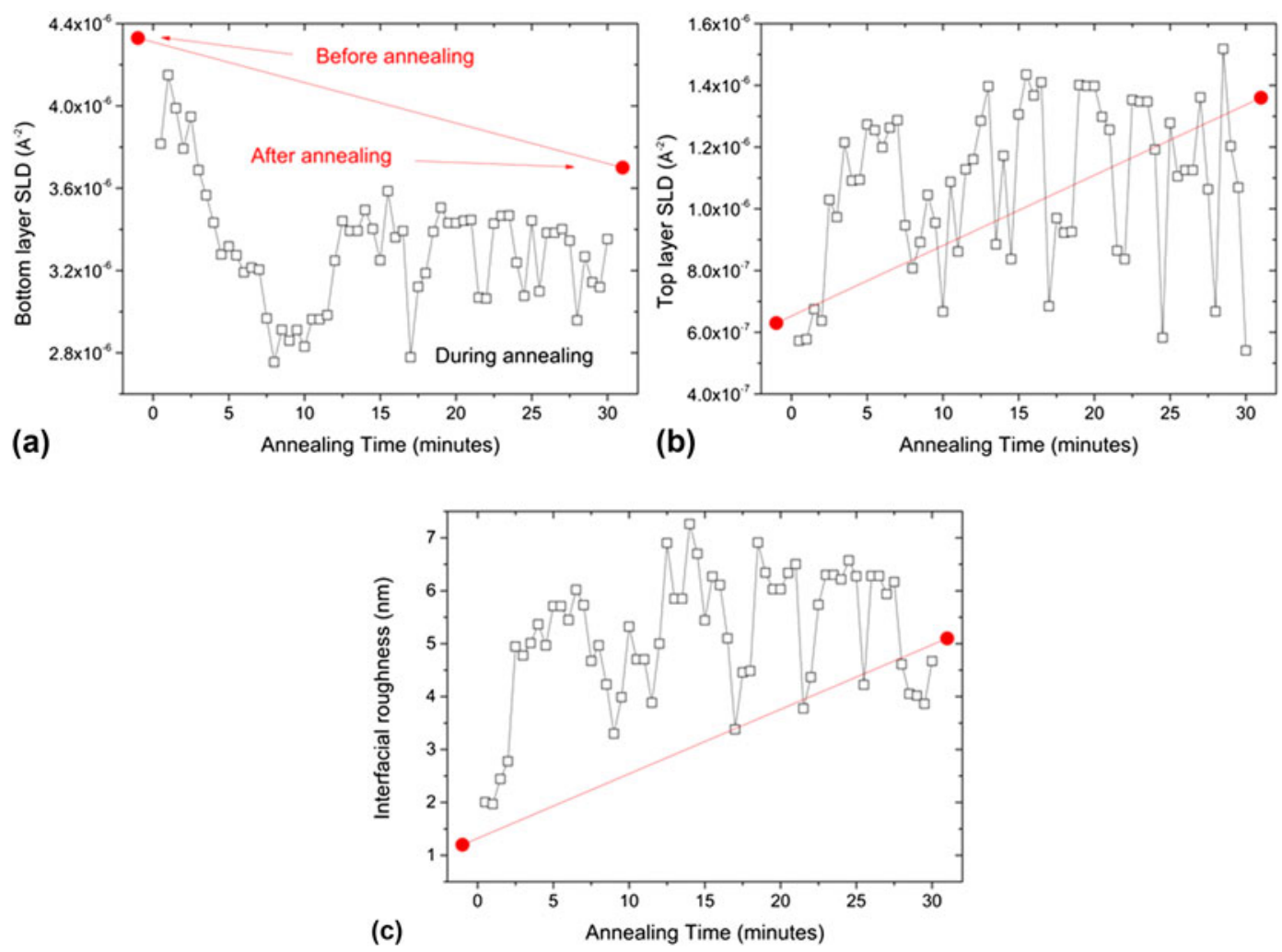

FIG. 4. Fit parameters for the PCBM $(\sim 20 \mathrm{~nm}) / \mathrm{P} 3 \mathrm{HT}(\sim 35 \mathrm{~nm})$ sample annealed in situ at $170{ }^{\circ} \mathrm{C}$. The reflectivity curves and SLD profiles for this sample are shown in Fig. 3. The filled circles correspond to fits of the full reflectivity curves before and after annealing the sample. The open squares correspond to fits of the reflectivity collected at a single incident angle during annealing (30 s collection time for each data point). The SLD of the bottom and top layers are shown in (a) and (b) respectively, and the interface roughness between these two layers is shown in (c).

top layers of $81 \%$ PCBM and 19\% PCBM respectively, and an interface roughness of $5.1 \mathrm{~nm}$.

The changes observed indicate that there has been a significant transfer of PCBM into the initially pure P3HT layer producing a P3HT-rich layer, and a transfer of P3HT into the initially pure PCBM layer producing a PCBM-rich layer, in a time frame of less than a few minutes. This is consistent with previous results in similar PCBM/P3HT bilayers. ${ }^{14,17}$ The migration of both species in this system can be contrasted with our findings for $\mathrm{PCBM}$ /polystyrene (PS) bilayers, using the same thickness PCBM (the same batch of material, spin-coated from the same solvent). ${ }^{26}$ In the simpler PCBM/PS system (in which only the PCBM can crystallize) mixing also occurred very rapidly, but here only the PCBM diffuses into the PS, with no PS migrating into the PCBM. The PCBM/PS system forms a consistent composition profile (between a PS-rich phase and a pure PCBM phase) that is suggestive of an equilibrium liquid-liquid interface (on the basis that the interface forms before any PCBM crystallizes, and is independent of annealing temperature and film thickness). The situation is very different for regioregular $\mathrm{P} 3 \mathrm{HT} / \mathrm{PCBM}$, as the as-cast polymer film contains extensive crystalline regions (see Supplementary Material Fig. S1 and previous studies ${ }^{31,34,35}$ ).
In our previous investigations of annealed PCBM $(20 \mathrm{~nm}) / \mathrm{PS}$ bilayers, we found a very strong influence of the polymer film thickness and temperature on the crystallization behavior of the PCBM, but no influence on the composition profile within the bilayer. ${ }^{26}$ To see how film thickness and temperature affect the mixing in the PCBM/P3HT system, bilayers with $20 \mathrm{~nm}$ of PCBM on the bottom and P3HT layers of various thicknesses on top, were annealed at $140{ }^{\circ} \mathrm{C}$. Figure 5 shows the reflectivity data and SLD profiles obtained from bilayer/ single layer fits with three different P3HT thicknesses, after ex situ annealing. As with the in situ measurements, migration of PCBM into the P3HT layer on annealing is evident for all three thicknesses of P3HT. A simple bilayer model fits the 8 and $35 \mathrm{~nm}$ P3HT sample data well [Figs. 5(a) and 5(b)]. For the $8 \mathrm{~nm}$ P3HT sample the best-fit has a PCBM-rich layer containing 90-98\% PCBM and a P3HT-rich layer containing 7-19\% PCBM. For the $35 \mathrm{~nm}$ P3HT sample the best-fit PCBM-rich layer contains 84-85\% PCBM and the P3HT-rich layer contains 18-20\% PCBM. The roughness of the buried PCBM-rich/P3HT-rich interface has best-fit values of 2.4-2.7 nm and 3.3-3.8 nm for the $8 \mathrm{~nm}$ P3HT and $35 \mathrm{~nm}$ P3HT samples respectively. The composition and interfacial roughness ranges quoted here refer to best 

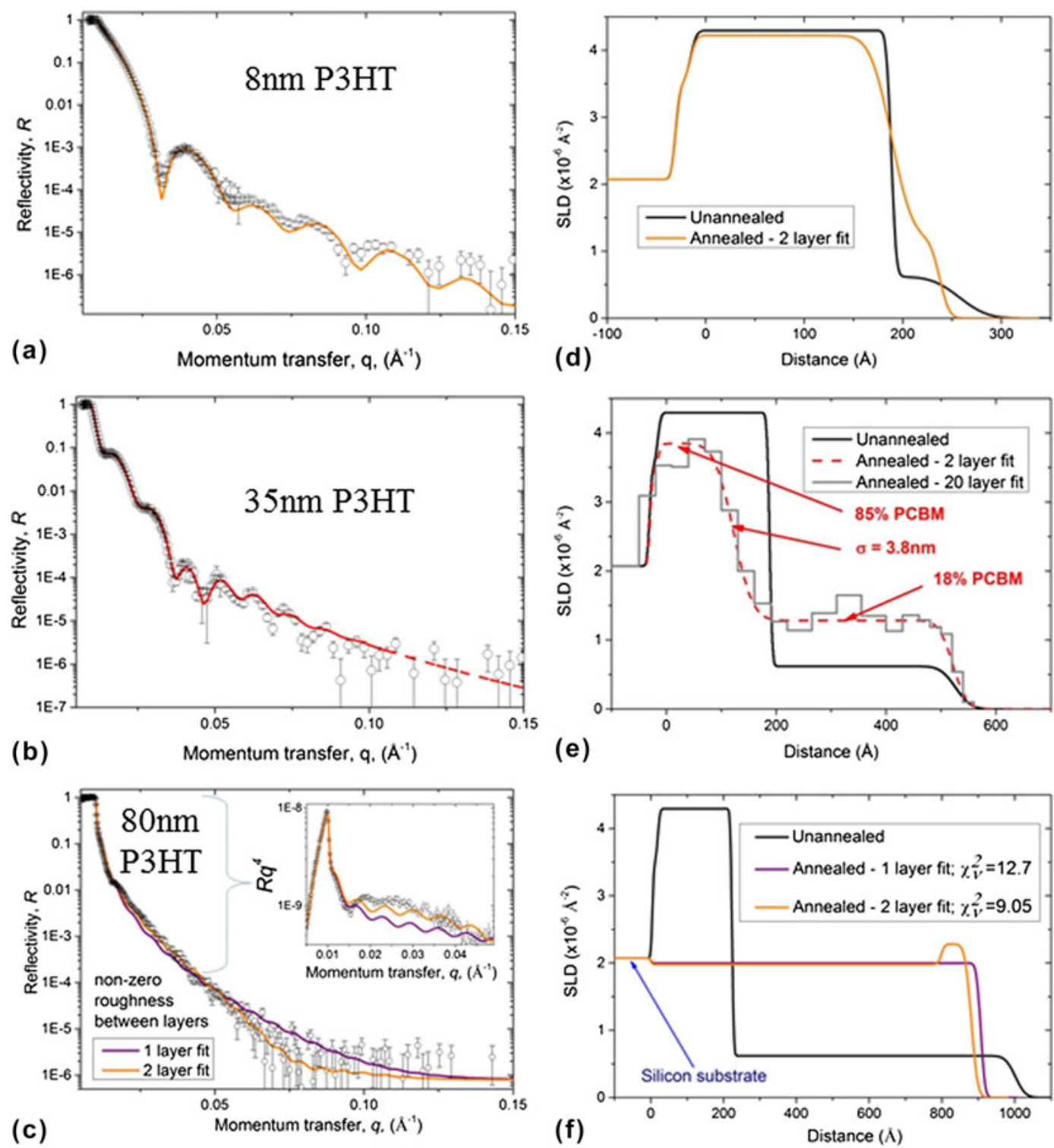

FIG. 5. Neutron reflectivity data and fits for PCBM $(20 \mathrm{~nm}) / \mathrm{P} 3 \mathrm{HT}$ bilayers on silicon, with three different thicknesses of P3HT. (a-c) Neutron reflectivity data and fits. All fits use either a single layer or bilayer model in which the thickness, SLD and roughness of each layer is allowed to vary. The SLD profiles corresponding to the best-fit lines in (a-c) are given in $(\mathrm{d}-\mathrm{f})$ respectively. Initial thicknesses of P3HT were approximately $8 \mathrm{~nm}$ in (a) and (d), $35 \mathrm{~nm}$ in (b) and (e) and $80 \mathrm{~nm}$ in (c) and (f). All samples were ex situ annealed at $140{ }^{\circ} \mathrm{C}$ (for $60 \mathrm{~min}$ for the 8 and $35 \mathrm{~nm}$ P3HT bilayers, and for $10 \mathrm{~min}$ for the $80 \mathrm{~nm}$ P3HT bilayer). A 20 layer fit to the $35 \mathrm{~nm}$ P3HT sample is also shown in (e); in this fit the interfacial roughness between all layers is set to zero, and the SLD of all layers is allowed to vary. There is no fixed oxide layer included in this model. There is also no fixed oxide layer included in the single/bilayer fits in (c) and (f). The unannealed SLD profiles in (d), (e) and (f) were calculated from AFM measurements (of the layer thicknesses and the surface roughness) of duplicate PCBM and P3HT single layers, and of the bilayer samples prior to annealing (imaging peripheral areas of the sample that were not irradiated by the neutron beam). The percentages (by volume) of PCBM in each layer and the roughness of the PCBM-rich/P3HT-rich interface, after annealing are given in (e) for the 2 layer fit to this sample. The inset in (c) shows the same data/fits presented in ' $R_{\mathrm{q}}^{4}$ ' format.

fits which were obtained by independent repeats of the data reduction and fitting methodology. The robustness of the fit parameters with-respect-to the inclusion/neglect of the oxide layer was also tested, and found to be negligible. It is important to point out that the PCBM concentration in the P3HT-rich (top) layer in the $8 \mathrm{~nm}$ P3HT sample is subject to significantly more uncertainty than the other layer concentrations in the fits of both the
8 and $35 \mathrm{~nm}$ P3HT samples, due to the fact that this sample consists of a very thin layer of low SLD material on top of a thicker layer of higher SLD. However, it is clear that significant mixing has occurred in this sample, as the data cannot be fitted well using pure P3HT and PCBM layer SLDs. A final test of the robustness of our modeling is provided by using an alternative approach to fit the data. Rather than using a bilayer model, with the 
gradient in SLD at the buried interface described by Gaussian roughness, a much larger number of discrete thin layers with adjustable SLD, but zero roughness between the layers is used to describe the SLD profile. The 20-layer fit in Fig. 5(e) shows a very similar SLD profile to the bilayer fit.

Our results show a close similarity between the reflectivity curves and fitted SLD profiles for $20 \mathrm{~nm}$ PCBM/35 nm P3HT bilayers after annealing at both $170{ }^{\circ} \mathrm{C}$ [Figs. 3(a) and 3(b)] and $140{ }^{\circ} \mathrm{C}$ [Figs. 5(b) and $5(\mathrm{e})]$. We now discuss this with regard to the state (crystalline/amorphous) of the PCBM and P3HT within the bilayers. Grazing-incidence X-ray diffraction (GIXD) measurements (see Supplementary Material Fig. S1) show extensive P3HT crystallization, but little evidence of significant PCBM crystallization on annealing at $140{ }^{\circ} \mathrm{C}$. GIXD measurements were not performed in parallel with the in situ neutron reflectivity measurements at $170{ }^{\circ} \mathrm{C}$. However, it is quite likely that some PCBM nanocrystallisation occurs at $170{ }^{\circ} \mathrm{C}$, if not on the timescale of the rapid mixing shown in Fig. 3, then within the 30 min annealing time (Leman et al. ${ }^{23}$ report crystallization of PCBM within a similar PCBM/P3HT bilayer, but with the PCBM layer on top, annealed at $160{ }^{\circ} \mathrm{C}$ for $10 \mathrm{~min}$ ). Given the strong temperature dependence of the crystallization of PCBM between 140 and $170{ }^{\circ} \mathrm{C},{ }^{34}$ and the high levels of crystalline material in spin-coated P3HT, ${ }^{31,34,35}$ the similar SLD profiles are what one would expect in a system where there is rapid diffusion of PCBM into the amorphous $\mathrm{P} 3 \mathrm{HT}$ regions, with little change in the fraction of amorphous P3HT and little influence of any PCBM crystallization that may occur at $170{ }^{\circ} \mathrm{C}$, during this rapid diffusion process. A similar lack of sensitivity to annealing temperature is found for PCBM/PS bilayers, but for a completely different reason. Migration of PCBM into PS also occurs within a few minutes and gives similar SLD profiles at both 140 and $170{ }^{\circ} \mathrm{C} .{ }^{26}$ However, in the case of PCBM/PS the composition profile is established in the absence of any crystallization, whereas in P3HT/PCBM the composition profile is likely to be largely determined by the volume fraction and micro/nano-structure of the pre-existing crystalline/amorphous regions within the polymer layer prior to bilayer annealing. ${ }^{31}$

For the $80 \mathrm{~nm}$ P3HT sample, the reflectivity curve is modeled reasonably well using a single layer fit (in which the SLD, thickness and roughness of the layer are allowed to vary), indicating that there is 'almost' complete mixing of PCBM and amorphous P3HT [see Figs. 5(c) and 5(f)]. However, this fit has an integrated SLD that is much too high (by a factor of $\sim 40 \%$; see Table SI in the Supplementary Material), given the known composition of the sample. Figure 5(f) also shows a bilayer fit for the $80 \mathrm{~nm}$ P3HT sample (in which the SLDs, thicknesses, and roughnesses of both layers are allowed to vary). This fit is visually better and has a lower value of the goodness-offit reduced chi-squared parameter, $\chi_{v}^{2},{ }^{36}$ compared to the single layer fit, but still has an integrated SLD that is unphysically high. Further approaches to fit the reflectivity from this sample were made using a number of different multilayer models. These approaches highlight the potential for nonuniqueness, in terms of different models that can adequately fit the data for the $80 \mathrm{~nm}$ P3HT sample, and hence place limits on the precision with which it is possible to describe the details of the composition profile in this sample. Discussion of these different approaches and the degree to which we are able to determine details of the composition profile is provided in the supplementary material (see Fig. S2 and accompanying discussion). The main conclusion from this discussion is that, despite the existence of significant uncertainty over the exact distribution of PCBM within the $80 \mathrm{~nm}$ P3HT sample, the physical picture represented by the reflectivity curve in Fig. 5(c) corresponds to a situation in which the entire PCBM bottom layer has mixed into the P3HT layer on top.

We also assessed the morphology of annealed bilayers using optical microscopy and AFM. This involved the samples used for the neutron reflectivity measurements and duplicate samples, annealed for various times at different temperatures. Fig. S3 shows the emergence of micron-sized PCBM crystals previously reported in bilayers annealed for 120 min. $^{26}$ No emergence of such crystals was seen in the samples used for neutron reflectivity (annealed for $60 \mathrm{~min}$ or less). Figure 6 shows typical surface morphology on the 35 and $80 \mathrm{~nm}$ P3HT neutron reflectivity samples annealed at $140{ }^{\circ} \mathrm{C}$. The morphology of the $35 \mathrm{~nm}$ P3HT sample consists of discrete features of around 10-25 nm in height separated by several microns or more. There is no sign of any dewetting on this sample (in contrast to the report by Ro et al. ${ }^{31}$ for P3HT/PCBM bilayers with the PCBM layer on top), and the root-mean-square (rms) roughness of the surface is small compared to the total bilayer thickness or the thickness of the P3HT layer (the maximum peak-to-trough height seen in profile 1 of Fig. 6(c) is also lower than the P3HT layer thickness). This negates the possibility that the reduction in the SLD of the bottom layer in Fig. 5(e) is due to lateral averaging of the SLD profile (averaged over the in-plane coherence length of the neutrons - tens of microns ${ }^{37,38}$ ) over regions that contain significant macroscopic (micron-sized) surface holes that penetrate into the bottom $20 \mathrm{~nm}$ of the sample. This is also the case for the $35 \mathrm{~nm}$ P3HT bilayer annealed in situ at $170{ }^{\circ} \mathrm{C}$; In comparison to the $35 \mathrm{~nm}$ P3HT sample, ex situ annealed at $140{ }^{\circ} \mathrm{C}$, this sample shows different 'dot-like' morphological features on the sample surface (see Fig. S4). We do not understand the reason for this morphological difference at present. 


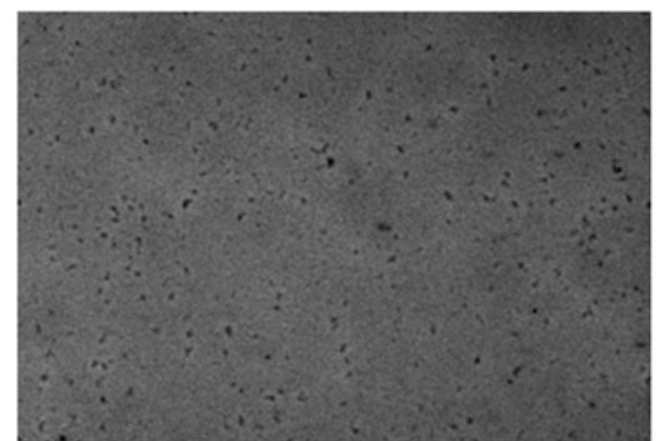

(a)
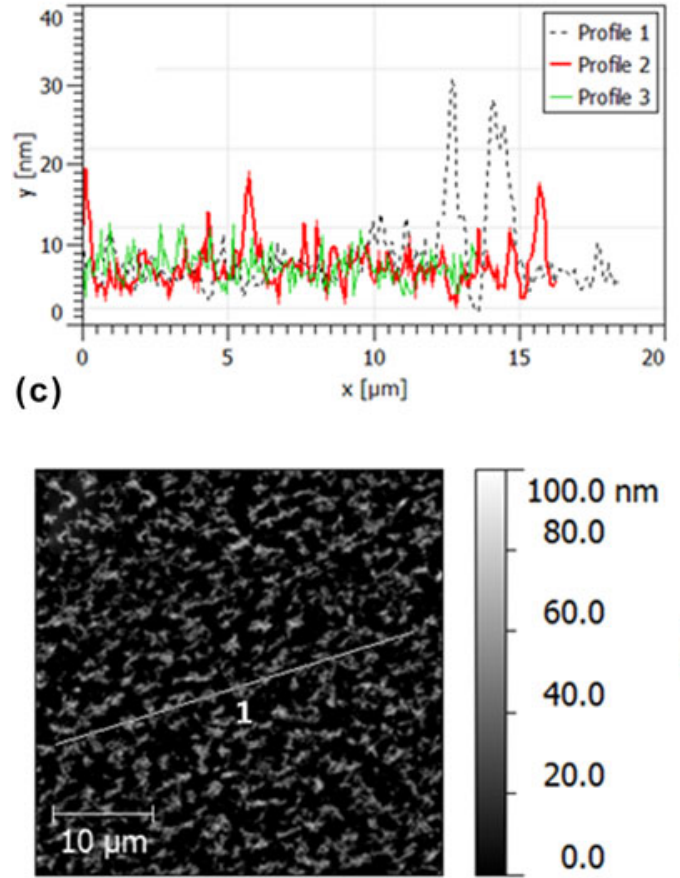

(e)
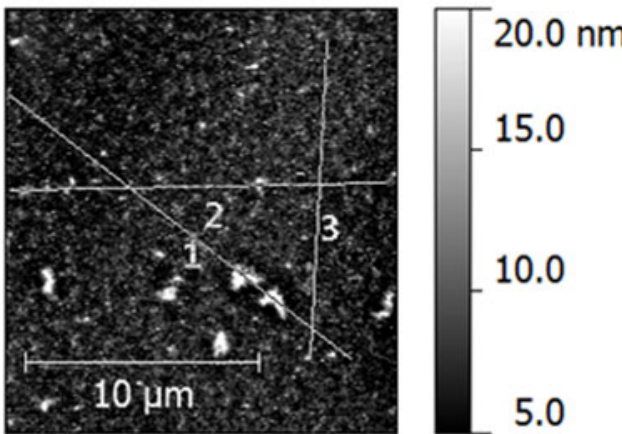

(b)

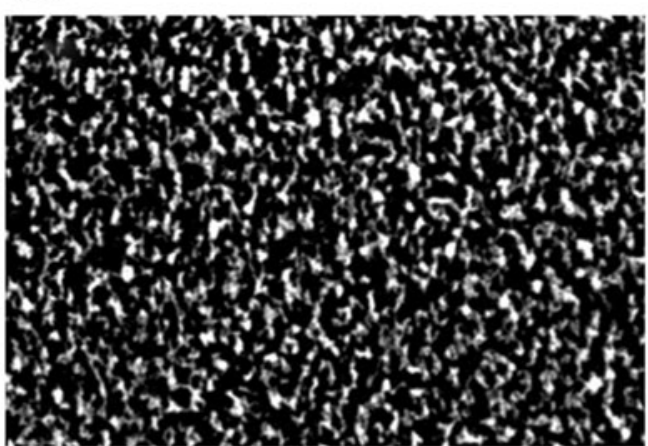

(d)

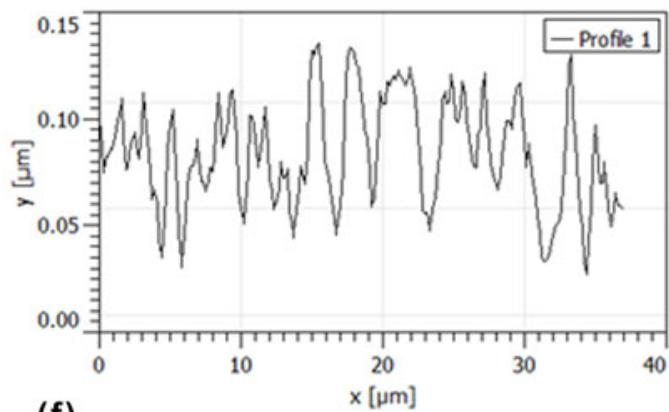

(f)

FIG. 6. Optical microscopy and atomic force microscopy (AFM) images of annealed (20 nm) PCBM/P3HT bilayers. (a) Optical microscopy image of a $35 \mathrm{~nm}$ P3HT bilayer annealed at $140{ }^{\circ} \mathrm{C}$ for $60 \mathrm{~min}$. (b) AFM image of the sample shown in (a). (c) Line profiles from the image shown in (b). (d) Optical microscopy of an $80 \mathrm{~nm}$ P3HT bilayer annealed at $140{ }^{\circ} \mathrm{C}$ for $10 \mathrm{~min}$. (e) AFM image of an $80 \mathrm{~nm}$ P3HT bilayer annealed at $140{ }^{\circ} \mathrm{C}$ for $60 \mathrm{~min}$. (f) A line profile from the image shown in (e). (a-d) were taken from the actual samples measured with neutron reflectivity, while (e) and (f) are from a duplicate of the sample show in (d), but annealed for $60 \mathrm{~min}$, rather than the $10 \mathrm{~min}$ (both AFM measurements and optical microscopy images on these two samples look very similar). The rms roughnesses of (b) and (e) are 2.7 and $26 \mathrm{~nm}$ respectively. AFM measurements (not shown) on the sample shown in (d) gave an rms roughness of $24 \pm 2 \mathrm{~nm}$ (averaged over five $40 \times 40 \mu \mathrm{m}$ scans from different parts of the sample). Images (a) and (d) are both of size $100 \times 68 \mu \mathrm{m}$.

We note that the volume fraction of PCBM in the bottom layers of the two $35 \mathrm{~nm}$ P3HT bilayer sample fits (81 and $85 \%$ ) are within the miscibility gap proposed for this system by Ulum et al. [Fig. 5(b)]. ${ }^{39}$ However more recent studies, ${ }^{23,40}$ including a study that looked at the impact of P3HT molecular weight on the Flory-Huggins interaction parameter (at $150{ }^{\circ} \mathrm{C}$ ) in this system ${ }^{40}$ concluded that, over the range of molecular weights used in the present study, the PCBM and P3HT were completely miscible. In this case the asymmetric composition profiles that we observe will result, not from any influence of liquid-liquid phase co-existence, but due to the extensive P3HT crystallisation that exists in the unannealed bilayers. It is possible that the pinning of amorphous P3HT chain sections at either one or more locations along the chain, by P3HT crystallites, restricts both the diffusion of polymer chains into the bottom layer and also PCBM molecules into the top layer of the sample. In the latter case, this is via a mechanism in which amorphous P3HT tie-chains, that bridge between crystallites, restrict ingress of PCBM into the amorphous $\mathrm{P} 3 \mathrm{HT}$ regions, as this requires swelling of the space between P3HT crystallites and a consequent reduction in the entropy of the tie chains. ${ }^{23}$ 
The situation is very different for the $80 \mathrm{~nm}$ P3HT bilayer annealed at $140{ }^{\circ} \mathrm{C}$. Figures 6(d)-6(f) show a much rougher sample surface for bilayers annealed for 10 and $60 \mathrm{~min}$. This is clearly the cause of the low reflectivity in Fig. 5(c). However, even here, fringes from the total thickness are still present (though very weak) and AFM again shows that the rough surface topography does not penetrate within $20 \mathrm{~nm}$ of the substrate. Therefore, although the relatively featureless reflectivity curve doesn't allow us to determine precise details regarding the composition profile of the sample, we propose that there has been close to full dissolution of the $20 \mathrm{~nm}$ bottom PCBM layer into the P3HT in this sample. Preservation of a distinct high-SLD layer of approximately $20 \mathrm{~nm}$ thickness at the substrate would give rise to significant fringes due to this layer, even in the presence of very large surface roughness [such as in Figs. 6(d)-6(f)], and significant interface roughness (see Fig. S6), and we therefore propose that such a layer does not exist. Finally, we note that a more definitive investigation of the composition profile within such a sample could, in principle, be performed by examining the off-specular scattering due to the lateral roughness in this sample. However, our previous experience in such off-specular analysis ${ }^{41}$ is that one requires off-specular data from a systematic series of samples, with much better counting statistics than those available in the present study.

Given that the $80 \mathrm{~nm}$ P3HT sample contains 20\% PCBM in total, and that the loading of PCBM within the P3HT-rich layer in the $35 \mathrm{~nm}$ P3HT samples at both 140 and $170{ }^{\circ} \mathrm{C}$ is $18-20 \%$, these findings are consistent with the previous report by $\mathrm{H}$. Chen et al. ${ }^{17}$ of a maximum PCBM volume fraction of around $20 \%$ within regioregular P3HT. However, we would like to also point out that the thickness dependence seen in Figs. 5 and S2 is qualitatively different to that seen by $\mathrm{H}$. Chen et al. In their study (in which the PCBM layer thickness, the P3HT layer thickness and the ratio of the two, all change from one sample to the next) $\mathrm{H}$. Chen et al. report more significant surface enrichment of PCBM (a larger surface excess) for samples containing a higher fraction of PCBM. In contrast, our results (for bilayers in which the fraction of PCBM in the sample is controlled by changing the P3HT thickness only) show no evidence for surface enrichment for the higher PCBM fraction $(\sim 36$ and $71 \%$ ) samples, with tentative evidence of possible surface enrichment by PCBM at a lower PCBM fraction $(\sim 20 \%)$ only.

Previous experiments with regiorandom and regioregular P3HT have demonstrated high levels of miscibility between PCBM and amorphous P3HT at 140 and $150{ }^{\circ} \mathrm{C}$, with the volume fraction of PCBM mixing into the P3HT layer within a regioregular-P3HT/PCBM bilayer being strongly influenced by the crystallinity in the P3HT layer prior to bilayer annealing. ${ }^{15,20,31}$ The similar PCBM loadings found in the present study at 140 and $170{ }^{\circ} \mathrm{C}$ lend further credence to this view, given the relatively modest $(\sim 10 \%)$ reported increase in crystalline P3HT content during bilayer annealing. ${ }^{31}$ After bilayer annealing (for up to $60 \mathrm{~s}$ ) Ro et al. ${ }^{31}$ find that the PCBM volume fraction within as-cast regioregular P3HT layers is approximately $40 \%$, while thermally pre-annealed P3HT layers only take up around 20\% PCBM. Approximate PCBM uptake levels of both $40^{14}$ and $20 \%^{17}$ are reported in other studies. However, pre-annealing of the P3HT is not performed in either of these studies, and so cannot account for this difference. The difference in PCBM uptake levels reported in the regioregular P3HT studies cannot also be attributed to different solvents used to deposit the P3HT layer; the studies by Ro et al. and $\mathrm{H}$. Chen et al. both use dichlorobenzene, while the present study uses toluene as the solvent for $\mathrm{P} 3 \mathrm{HT}$ and finds similar uptake levels to $\mathrm{H}$. Chen et al. ${ }^{17} \mathrm{D}$. Chen et al. $^{15}$ also report nonuniform PCBM and P3HT distributions in annealed bilayers (fabricated from PCBM and as-cast regioregular P3HT) with a total PCBM content of $38 \%$. It is therefore not completely clear at present why there is such a range of composition profiles found across various studies. Ro et al. ${ }^{31}$ propose that the P3HT microstructure, as well as the crystalline fraction, are likely to be key, and one would expect that this might be significantly influenced by the degree of regioregularity or the molecular weight distribution of the P3HT. However, one other possible factor could be the thickness of either the PCBM or polymer layer. There is significant variation in layer thicknesses across the studies performed to-date, and our recent work on model PCBM/PS and PCBM/P3HT bilayers has shown that there is a strong dependence of both the PCBM and P3HT crystal microstructure and nanostructure on the thickness of both layers. ${ }^{26}$

\section{CONCLUSION}

In situ and ex situ neutron reflectivity has been used to characterize annealed regioregular-P3HT/PCBM bilayers on silicon, as a function of annealing temperature and P3HT layer thickness. This reveals mixing of PCBM and $\mathrm{P} 3 \mathrm{HT}$ at a similar level to that reported by some previous workers. Examination of in situ annealed samples has allowed us to monitor the rapid development of mixing. Careful analysis of ex situ annealed samples has allowed us to robustly evaluate the evidence for the formation of composition profiles that can be parameterized as simple bilayers or as more complex profiles. We also propose that the potential interplay between layer thickness and crystal microstructure/nanostructure should lead to careful control/consideration of layer thicknesses, when performing comparative studies. 


\section{ACKNOWLEDGMENTS}

We thank the ILL for the award of beam time (proposal number 9-11-1519), and the staff of D17 for help during the experiment. We also thank J. Emyr Macdonald, Mark Hampton, Jonathan Rawle and the staff of beam-line IO7 at Diamond Light Source for their support during the GIXD experiments. AH and DM acknowledge Huw Summers for use of his optical microscope and Chris Wright for use of his AFMs. DM acknowledges EPSRC for funding his studentship via the Doctoral Training Grant to Swansea University (Grant No. EP/P505763/1).

\section{REFERENCES}

1. F.C. Krebs: Fabrication and processing of polymer solar cells: A review of printing and coating techniques. Sol. Energy Mater. Sol. Cells 93, 394 (2009).

2. C.J. Brabec, S. Gowrisanker, J.J.M. Halls, D. Laird, S. Jia, and S.P. Williams: Polymer-fullerene bulk-heterojunction solar cells. Adv. Mater. 22, 3839 (2010).

3. F. Guo, T. Ameri, K. Forberich, and C.J. Brabec: Semitransparent polymer solar cells. Polym. Int. 62, 1408 (2013).

4. F.C. Krebs, M. Jørgensen, K. Norrman, O. Hagemann, J. Alstrup, T.D. Nielsen, J. Fyenbo, K. Larsen, and J. Kristensen: A complete process for production of flexible large area polymer solar cells entirely using screen printing-First public demonstration. Sol. Energy Mater. Sol. Cells 93, 422 (2009).

5. F. Liu, Y. Gu, J.W. Jung, W.H. Jo, and T.P. Russell: On the morphology of polymer-based photovoltaics. J. Polym. Sci., Part B: Polym. Phys. 50, 1018 (2012).

6. M.T. Dang, L. Hirsch, and G. Wantz: P3HT:PCBM, best seller in polymer photovoltaic research. Adv. Mater. 23, 3597 (2011).

7. S. Günes, H. Neugebauer, and N.S. Sariciftci: Conjugated polymer-based organic solar cells. Chem. Rev. 107, 1324 (2007).

8. Z. Li, K. Ho Chiu, R. Shahid Ashraf, S. Fearn, R. Dattani, H. Cheng Wong, C-H. Tan, J. Wu, J.T. Cabral, and J.R. Durrant: Toward improved lifetimes of organic solar cells under thermal stress: Substrate-dependent morphological stability of PCDTBT: PCBM films and devices. Sci. Rep. 5, 15149 (2015).

9. A.J. Parnell, A.D.F. Dunbar, A.J. Pearson, P.A. Staniec, A.J.C. Dennison, H. Hamamatsu, M.W.A. Skoda, D.G. Lidzey, and R.A.L. Jones: Depletion of PCBM at the cathode interface in P3HT/PCBM thin films as quantified via neutron reflectivity measurements. Adv. Mater. 22(22), 2444 (2010).

10. G. Dennler, M.C. Scharber, and C.J. Brabec: Polymer-fullerene bulk-heterojunction solar cells. Adv. Mater. 21, 1323 (2009).

11. K.H. Lee, P.E. Schwenn, A.R.G. Smith, H. Cavaye, P.E. Shaw, M. James, K.B. Krueger, I.R. Gentle, P. Meredith, and P.L. Burn: Morphology of all-solution-processed "bilayer" organic solar cells. Adv. Mater. 23, 766 (2010).

12. P. Westacott, J.R. Tumbleston, S. Shoaee, S. Fearn, J.H. Bannock, J.B. Gilchrist, S. Heutz, J. deMello, M. Heeney, H. Ade, J. Durrant, D.S. McPhail, and N. Stingelin: On the role of intermixed phases in organic photovoltaic blends. Energy Environ. Sci. 6(9), 2756 (2013).

13. A.A. Herzing, H.W. Ro, C.L. Soles, and D.M. DeLongchamp: Visualization of phase evolution in model organic photovoltaic structures via energy-filtered transmission electron microscopy. ACS Nano 7(9), 7937 (2013).

14. N.D. Treat, M.A. Brady, G. Smith, M.F. Toney, E.J. Kramer, C.J. Hawker, and M.L. Chabinyc: Interdiffusion of PCBM and P3HT reveals miscibility in a photovoltaically active blend. $A d v$. Energy Mater. 1, 82 (2011).
15. D. Chen, A. Nakahara, D. Wei, D. Nordlund, and T.P. Russell: P3HT/PCBM bulk heterojunction organic photovoltaics: Correlating efficiency and morphology. Nano Lett. 11, 561 (2011).

16. R.C. Nieuwendaal, H.W. Ro, D.S. Germack, R.J. Kline, M.F. Toney, C.K. Chan, A. Agrawal, D. Gundlach, D.L. VanderHart, and D.M. Delongchamp: Measuring domain sizes and compositional heterogeneities in P3HT-PCBM bulk heterojunction thin films with $1 \mathrm{H}$ spin diffusion NMR spectroscopy. Adv. Funct. Mater. 22, 1255 (2012).

17. H. Chen, R. Hegde, J. Browning, and M.D. Dadmun: The miscibility and depth profile of PCBM in P3HT: Thermodynamic information to improve organic photovoltaics. Phys. Chem. Chem. Phys. 14, 5635 (2012).

18. A. Tada, Y. Geng, Q. Wei, K. Hashimoto, and K. Tajima: Tailoring organic heterojunction interfaces in bilayer polymer photovoltaic devices. Nat. Mater. 10, 450 (2011).

19. V.S. Gevaerts, L.J.A. Koster, M.M. Wienk, and R.A.J. Janssen: Discriminating between bilayer and bulk heterojunction polymer: fullerene solar cells using the external quantum efficiency. ACS Appl. Mater. Interfaces 3, 3252 (2011).

20. D. Chen, F. Liu, C. Wang, A. Nakahara, and T.P. Russell: Bulk heterojunction photovoltaic active layers via bilayer interdiffusion. Nano Lett. 11, 2071 (2011).

21. A.J. Clulow, A. Armin, K.H. Lee, A.K. Pandey, C. Tao, M. Velusamy, M. James, A. Nelson, P.L. Burn, I.R. Gentle, and P. Meredith: Determination of fullerene scattering length density: A critical parameter for understanding the fullerene distribution in bulk heterojunction organic photovoltaic devices. Langmuir 30(5), 1410 (2014)

22. A.M. Higgins, M. Sferrazza, R.A. Jones, P.C. Jukes, J.S. Sharp, L.E. Dryden, and J. Webster: The timescale of spinodal dewetting at a polymer/polymer interface. Eur. Phys. J. Soft Matter 8(2), 137 (2002).

23. D. Leman, M.A. Kelly, S. Ness, S. Engmann, A. Herzing, C. Snyder, H.W. Ro, R.J. Kline, D.M. DeLongchamp, and L.J. Richter: In situ characterization of polymer-fullerene bilayer stability. Macromolecules 48(2), 383 (2015).

24. T.A.M. Ferenczi, J. Nelson, C. Belton, A.M. Ballantyne, M. Campoy-Quiles, F.M. Braun, and D.D.C. Bradley: Planar heterojunction organic photovoltaic diodes via a novel stamp transfer process. J. Phys.: Condens. Matter 20(47), 475203 (2008).

25. R.A.L. Jones and R.W. Richards: Polymers at Surfaces and Interfaces, 1st ed. (Cambridge University Press, Cambridge, UK, 1999).

26. D. Môn, A.M. Higgins, D. James, M. Hampton, J.E. Macdonald, M.B. Ward, P. Gutfreund, S. Lilliu, and J. Rawle: Bimodal crystallization at polymer-fullerene interfaces. Phys. Chem. Chem. Phys. 17(3), 2216 (2015).

27. R. Cubitt and G. Fragneto: D17: The new reflectometer at the ILL. Appl. Phys. A: Mater. Sci. Process. 74, S329 (2002).

28. A. Nelson: Co-refinement of multiple contrast neutron/X-ray reflectivity data using MOTOFIT. J. Appl. Crystallogr. 29, 273276 (2006)

29. NIST: Neutron activation and scattering calculator. Available at: www.ncnr.nist.gov/resources/activation/ (accessed August 2016)

30. C.S. Lee and M.D. Dadmun: Important thermodynamic characteristics of poly(3-hexyl thiophene). Polymer 55(1), 4 (2014).

31. H.W. Ro, B. Akgun, B.T.O. Connor, M. Hammond, R.J. Kline, C.R. Snyder, S.K. Satija, A.L. Ayzner, M.F. Toney, C.L. Soles, and D.M. Delongchamp: Poly(3-hexylthiophene) and [6,6]-phenyl$\mathrm{C}_{61}$-butyric acid methyl ester mixing in organic solar cells. Macromolecules 45, 6587 (2012).

32. J.S. Higgins and H.C. Benoit: Polymers \& Neutron Scattering, 1st ed. (Oxford University Press, Oxford, UK, 1994). 
33. R-J. Roe: Methods of X-ray and Neutron Scattering in Polymer Science, 1st ed. (Oxford University Press, Oxford, UK, 2000).

34. E. Verploegen, R. Mondal, C.J. Bettinger, S. Sok, M.F. Toney, and Z. Bao: Effects of thermal annealing upon the morphology of polymer-fullerene blends. Adv. Funct. Mater. 20(20), 3519 (2010).

35. P.E. Hopkinson, P.A. Staniec, A.J. Pearson, A.D.F. Dunbar, T. Wang, A.J. Ryan, R.A.L. Jones, D.G. Lidzey, and A.M. Donald: A phase diagram of the P3HT:PCBM organic photovoltaic system: Implications for device processing and performance. Macromolecules 44(8), 2908 (2011).

36. I.G. Hughes and T.P.A. Hase: Measurements and Their Uncertainties, 1st ed. (Oxford University Press, Oxford, UK, 2010).

37. R.M. Richardson, J.R.P. Webster, and A. Zarbakhsh: Study of offspecular neutron reflectivity using a model system. J. Appl. Crystallogr. 30(6), 943 (1997).
38. T.P. Russell: X-ray and neutron reflectivity for the investigation of polymers. Mater. Sci. Rep. 5(4), 171 (1990).

39. S. Ulum, N. Holmes, M. Barr, A.L.D. Kilcoyne, B.B. Gong, X. Zhou, W. Belcher, and P. Dastoor: The role of miscibility in polymer:fullerene nanoparticulate organic photovoltaic devices. Nano Energy 2(5), 897 (2013).

40. F. Liu, D. Chen, C. Wang, K. Luo, W. Gu, A.L. Briseno, J.W. Hsu, and T.P. Russell: Molecular weight dependence of the morphology in P3HT:PCBM solar cells. ACS Appl. Mater. Interfaces 6(22), 19876 (2014)

41. D. James, A.M. Higgins, P. Rees, M. Geoghegan, M.R. Brown, S-S. Chang, D. Môn, R. Cubitt, R. Dalgliesh, and P. Gutfreund: Measurement of molecular mixing at a conjugated polymer interface by specular and off-specular neutron scattering. Soft Matter 11(48), 9393 (2015).

\section{Supplementary Material}

To view supplementary material for this article, please visit https://doi.org/10.1557/jmr.2017.59. 